OPEN ACCESS

Edited by:

Oscar Arias-Carrión,

Hospital General Dr. Manuel Gea

Gonzalez, Mexico

Reviewed by:

Gennaro Pagano,

King's College London,

United Kingdom

José M. Salas-Pacheco,

Universidad Juárez del Estado de

Durango, Mexico

*Correspondence:

Hiroshi Kataoka

hk55@naramed-u.ac.jp

Specialty section:

This article was submitted to

Movement Disorders,

a section of the journal

Frontiers in Neurology

Received: 16 February 2017

Accepted: 27 September 2017

Published: 16 October 2017

Citation:

Kataoka H, Sawada Y, Namizaki T,

Shimozato N, Yoshiji $H$ and Ueno S

(2017) Intrajejunal Infusion of

Levodopa-Carbidopa Gel Can

Continuously Reduce the

Severity of Dropped Head in

Parkinson's Disease.

Front. Neurol. 8:547.

doi: 10.3389/fneur.2017.00547

\section{Intrajejunal Infusion of}

Levodopa-Carbidopa Gel Can Continuously Reduce the Severity of Dropped Head in Parkinson's Disease

\author{
Hiroshi Kataoka1*, Yasuhiko Sawada², Tadashi Namizaki², Naotaka Shimozato², \\ Hitoshi Yoshiji ${ }^{2}$ and Satoshi Ueno ${ }^{1}$ \\ ${ }^{1}$ Department of Neurology, Nara Medical University, Kashihara, Japan, ${ }^{2}$ Third Department of Internal Medicine, \\ Nara Medical University, Kashihara, Japan
}

Dropped head can occur in patients with Parkinson's disease and make their quality of life unpleasant because they cannot obtain a frontal view. The pathophysiologic involvement of dopamine agonist or central or peripheral mechanisms has been proposed. Levodopa therapy with the withdrawal of dopamine agonists was sometimes effective, but the effect in most patients did not persist for the entire day. We describe a patient with Parkinson's disease whose dropped head responded throughout the day to the continuous intrajejunal infusion of levodopa-carbidopa intestinal gel (LCIG). During off-periods before treatment with $\mathrm{LCIG}$, severe akinesia and freezing of gait were evident, and she could not continuously obtain a frontal view because of the dropped head. About 20 min after the intrajejunal infusion of LCIG, these features remarkably improved, and she could obtain a frontal view. The angle of dropped head was improved from 39.39 to $14.04^{\circ}$. This case suggests that infusion of LCIG can reduce the severity of dropped head for a longer period than oral levodopa.

Keywords: Parkinson, levodopa-carbidopa intestinal gel, dropped head, antecollis, intrajejunal infusion

\section{INTRODUCTION}

Dropped head can occur in patients with Parkinson's disease and make their quality of life unpleasant because they cannot obtain a frontal view. The pathophysiologic involvement of dopamine agonist or central or peripheral mechanisms has been proposed (1). Levodopa therapy with the withdrawal of dopamine agonists was sometimes effective, but the effect in most patients did not persist for the entire day. We describe a patient with Parkinson's disease whose dropped head responded throughout the day to the continuous intrajejunal infusion of levodopa-carbidopa intestinal gel (LCIG).

\section{CASE REPORT}

Eleven years ago, a 72-year-old right-handed woman noticed left-hand tremor. In 2006, she consulted a hospital physician, and cogwheel rigidity, resting tremor in the right hand, and akinesia 
were present. These features responded to treatment with oral levodopa (200 mg/day). In February 2008, she received levodopa (200 mg/day) and pramipexole $(0.5 \mathrm{mg} /$ day). She showed signs of moderate parkinsonism, including resting tremor in the right hand, right-dominant rigidity in all four limbs, and akinesia. In August 2009, the dose of pramipexole was increased to $1 \mathrm{mg} /$ day because of the resting tremor. She complained that her trunk became bent, and lumbar pain was evident. She showed mild-to-moderate trunk flexion. Pramipexole was discontinued, and the symptoms resolved. Since 2010, the severity of akinesia increased, and gait difficulty gradually developed. In June 2014, she received levodopa (300 mg/day), selegiline (10 mg/day), and zonisamide (50 mg/day). In May 2015, wearing-off was evident, and the dose of levodopa was increased to $400 \mathrm{mg} /$ day. In October, peak-dose dyskinesia was evident, and zonisamide was withdrawn. In April 2016, wearing off and troublesome dyskinesia developed. She also noticed painful lower abdominal contractions several times during off-periods in the night. Treatment with zonisamide (25 mg/day) was therefore resumed, but the lower abdominal contractions did not resolve. In October, she noticed dropped head while receiving a stable dosage of levodopa (400 mg/day), selegiline (10 mg/day), and zonisamide ( $25 \mathrm{mg} /$ day). Since two phenotypes of levodopa-responsiveness exist in patients with abnormal posture including dropped head (2), we performed intravenous dopamine challenge testing. The intravenous infusion of levodopa (50 $\mathrm{mg}$ ) reduced the severity of dropped head, and the dose of levodopa was therefore increased to $500 \mathrm{mg} /$ day. The severity of dropped head mildly decreased. In early November, the dose of zonisamide was increased (50 mg/day) because of prolonged off-periods, and subsequently rotigotine ( $9 \mathrm{mg} /$ day) was begun. In the middle of November, dropped head redeveloped. Rotigotine was discontinued, and the dose of levodopa was increased to $600 \mathrm{mg} /$ day (in 3 divided doses per day) while continuing stable doses of selegiline (10 mg/day) and zonisamide $(50 \mathrm{mg} /$ day). Dropped head and wearing-off were unchanged, and dyskinesia further developed. She was admitted to our hospital in December. The frequency of levodopa treatment was increased to five times per day. The dyskinesia mildly decreased, but off-periods in the morning, evening, and night were unchanged. Painful lower abdominal contractions also occurred from the evening to night. The dropped head required the patient to wear a neck collar to obtain a frontal view all day. In December, she received LCIG via a nasoduodenal tube. She had no history of psychosis or hallucinations. The Mini-Mental Score Examination score was 26. Dropped head was evaluated by taking photographs of the lateral aspects of the head, both at rest and during the patient's best effort to avert her neck. The angle of dropped head was calculated with the use of "Image J" software (3) by drawing a line between the vertex $(\mathrm{Cz})$ and the seventh spinous process, as described previously (2) (Figure 1).

During off-periods before treatment with LCIG, severe akinesia and freezing of gait were evident, and she could not continuously obtain a frontal view because of the dropped head (Videos S1 and S2 in Supplementary Material). The severity of dropped head increased during off-periods and mildly decreased during on-periods. About $20 \mathrm{~min}$ after
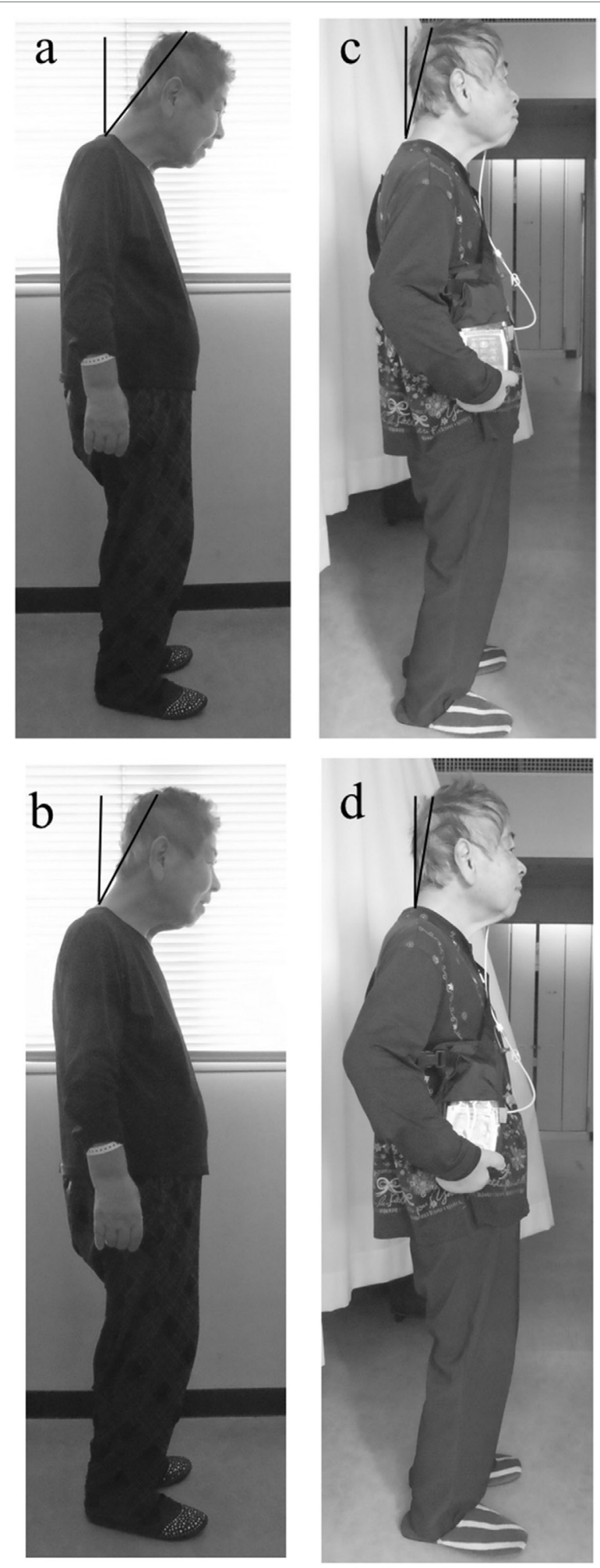

FIGURE 1 | A patient with dropped head before (A,B) and after (C,D) continuous intrajejunal infusion of levodopa-carbidopa intestinal gel (LCIG). After infusion of LCIG, the angle of antecollis was reduced in a natural position (A-C) and in a position with the neck averted with maximum effort (B-D). The black line is located between the vertex (Cz) and seventh spinous process. 
the intrajejunal infusion of LCIG, these features remarkably improved, and she could obtain a frontal view (Videos S3 and S4 in Supplementary Material). The Hoehn-Yahr stage decreased from 4 to 2, and balance also improved. The axial sum scores (items 8, 12, and 13) on the new revised Unified Parkinson's Disease Rating Scale (UPDRS)-III (4) decreased from 9 to 1. The score on part III of the revised UPDRS decreased from 69 to 11 .

The angle of dropped head was $39.39^{\circ}$ before the infusion of LCIG (Figure 1A). On the day when the infusion of LCIG was begun, the severity of dropped head improved enough for the patient to obtain a frontal view. From day 5 after starting the LCIG infusion, the dose of LCIG (levodopa/carbidopa: $22 / 5.5 \mathrm{mg} / \mathrm{h}$ ) was not changed. Seven days after starting LCIG, we repeatedly examined the angle of the dropped head. The angle in the natural position improved to $14.04^{\circ}$ (Figure 1C). On maximum effort to avert the neck, the angle decreased from 28.17 to $9.73^{\circ}$ (Figures 1B,D). This good status was maintained until the evening. After the evening on the same day, the angle deteriorated to $31.53^{\circ}$ in the natural position and $22.62^{\circ}$ on maximum effort to avert the neck. However, these angles were less than those before the infusion of LCIG. These serial evaluations were performed during on-periods, and the Hoehn-Yahr stage was 2 during all evaluations. The score of dyskinesia on items 1 and 2 of part IV of the newly revised UPDRS (4) decreased from 4 and 3 to 1 and 1 , respectively. The patient did not complain of painful abdominal contractions. This condition was maintained for 1 day after the second evaluation. On the first night, she had transient visual hallucinations only during the infusion of LCIG (levodopa/carbidopa: 28/7 mg/h). Written informed consent was obtained from the patient for the publication of this case report.

\section{DISCUSSION}

Treatment with oral levodopa improves head position, but this finding was inconsistent previously (1). In our study, dropped head rapidly responded to LCIG, and this response to levodopa persisted for a prolonged time. LCIG has not been previously reported to be effective against dropped head or axial symptoms. Doherty et al. mentioned that antecollis might be an off-state phenomenon or a dyskinesia-like phenomenon related to dopaminergic medications (1). Recently, we studied patients with PD who had anterior trunk flexion, dropped head, or lateral trunk flexion and investigated whether abnormal posture responded to levodopa by means of intravenous dopamine challenge testing (2). In patients with abnormal postures related to the "off" state, the change in the angle was significantly greater than that of patients whose abnormal posture was not related to the "off" state. This observation was also seen in patients with dropped head (2). Our patient also had dyskinesia and mild fluctuations of dropped head, and the dropped head in the present patient responded to intravenous dopamine challenge testing. Intermittent intake of oral levodopa leads to fluctuating plasma levodopa levels due to erratic gastric emptying and variable jejunal absorption. This variability in plasma levodopa concentrations leads to nonphysiologic intermittent or pulsatile stimulation of dopamine receptors, which can cause wearing-off or dyskinesia, especially in a dopamine-depleted state (5). As for bioavailability, LCIG results in approximately 50\% lower variability in levodopa concentrations than does oral administration of levodopa-carbidopa (6). This leads to shorter off-periods or milder dyskinesia. In addition, LCIG was shown to be absorbed faster than oral levodopa in a pharmacokinetics study in patients with advanced Parkinson's disease (6). Rapid absorption in addition to stable delivery of levodopa might be responsible for the prolonged response of dropped head to levodopa.

\section{CONCLUSION}

After infusion of LCIG, dropped head in our patient deteriorated after the evening, and the ability to maintain a good status of dropped head appeared to be limited. However, this case suggests that infusion of LCIG can reduce the severity of dropped head for a longer period than oral levodopa.

\section{ETHICS STATEMENT}

No investigations or interventions were performed outside of routine clinical care for this patient. As this is a case report, without experimental intervention into routine care, no formal research ethics approval was required. Written, fully informed consent was received from the patient. This case study reports routine clinical care provided for a patient only.

\section{AUTHOR CONTRIBUTIONS}

HK was responsible for the overall study design and contributed to analysis and interpretation of the data and wrote the manuscript. HK, YS, TN, and NS contributed to running the study and the acquisition of data. HK, HY, and SU contributed to drafting and critical revision of part of the submitted materials.

\section{FUNDING}

HK received JSPS KAKENHI Grant Number (15K9356).

\section{SUPPLEMENTARY MATERIAL}

The Supplementary Material for this article can be found online at http://www.frontiersin.org/article/10.3389/fneur.2017.00547/ full\#supplementary-material.

VIDEOS S1 AND S2 | The severity of Parkinson's disease before the continuous intrajejunal infusion of levodopa-carbidopa intestinal gel.

VIDEOS S3 AND S4 | The severity of Parkinson's disease after the continuous intrajejunal infusion of levodopa-carbidopa intestinal gel. 


\section{REFERENCES}

1. Doherty KM, van de Warrenburg BP, Peralta MC, Silveira-Moriyama L, Azulay JP, Gershanik OS, et al. Postural deformities in Parkinson's disease. Lancet Neurol (2011) 10:538-49. doi:10.1016/S1474-4422(11)70067-9

2. Kataoka H, Ueno S. Can postural abnormality really respond to levodopa in Parkinson's disease? J Neurol Sci (2017) 377:179-84. doi:10.1016/j.jns.2017. 04.025

3. Schneider CA, Rasband WS, Eliceiri W. NIH Image to ImageJ: 25 years of image analysis. Nat Methods (2012) 9:671-5. doi:10.1038/nmeth.2089

4. Goetz CG, Tilley BC, Shaftman SR, Stebbins GT, Fahn S, Martinez-Martin P, et al. Movement Disorder Society-sponsored revision of the Unified Parkinson's Disease Rating Scale (MDS-UPDRS): scale presentation and clinimetric testing results. Mov Disord (2008) 23:2129-70. doi:10.1002/mds.22340

5. Olanow CW, Kieburtz K, Odin P, Espay AJ, Standaert DG, Fernandez HH, et al. Continuous intrajejunal infusion of levodopa-carbidopa intestinal gel for patients with advanced Parkinson's disease: a randomised, controlled, double-blind, double-dummy study. Lancet Neurol (2014) 13:141-9. doi:10.1016/ S1474-4422(13)70293-X

6. Othman AA, Dutta S. Population pharmacokinetics of levodopa in subjects with advanced Parkinson's disease: levodopa-carbidopa intestinal gel infusion vs. oral tablets. Br J Clin Pharmacol (2014) 78:94-105. doi:10.1111/bcp.12324

Conflict of Interest Statement: The authors declare that the research was conducted in the absence of any commercial or financial relationships that could be construed as a potential conflict of interest.

Copyright (c) 2017 Kataoka, Sawada, Namizaki, Shimozato, Yoshiji and Ueno. This is an open-access article distributed under the terms of the Creative Commons Attribution License (CC BY). The use, distribution or reproduction in other forums is permitted, provided the original author(s) or licensor are credited and that the original publication in this journal is cited, in accordance with accepted academic practice. No use, distribution or reproduction is permitted which does not comply with these terms. 